\title{
Long-term clinical outcome in patients with acute coronary syndrome and dysglycaemia
}

\author{
Jeanette Kuhl ${ }^{1,2^{*} \mathbb{D}}$, Gun Jörneskog ${ }^{3}$, Malin Wemminger ${ }^{1}$, Mattias Bengtsson ${ }^{1}$, Pia Lundman ${ }^{1}$ and Majid Kalani ${ }^{1}$
}

\begin{abstract}
Background: Diabetes and impaired glucose tolerance (IGT) are major risk factors for atherosclerosis including coronary artery disease (CAD). The present study's aim was to investigate the importance of glucose tolerance for longterm clinical outcome in patients with acute coronary syndrome (ACS).
\end{abstract}

Methods: A total 1062 consecutive patients, 781 men and 281 women, aged 32-80 years, admitted to the coronary care unit at Danderyd University Hospital, Stockholm, for ACS from 2006 to 2008 were included. At discharge, the patients were categorized according to an oral glucose tolerance test (OGTT) as having normal glucose tolerance (NGT), $n=295$ (28\%); impaired fasting glucose (IFG) and IGT, $n=299$ (28\%); diabetes discovered by OGTT, $n=156$ (15\%); or known diabetes at admission, $\mathrm{n}=312(29 \%)$. Mortality and reinfarction rates were studied during a mean follow-up time of $4.0( \pm 0.8)$ years. Clinical outcome data were obtained from the Swedish Coronary Angiography and Angioplasty Registry and the Swedish National Registry.

Results: There was significantly higher $(p<0.001)$ mortality within, 30 days, 1 and 3 years in patients with known diabetes as compared to the other groups. During the follow-up, 86 patients ( $28 \%)$ with known diabetes had reinfarction as compared to 36 patients (12\%) with NGT and 79 patients (17\%) with dysglycaemia (IFG, IGT and diabetes) discovered by OGTT.

Conclusion: A majority (72\% in this study) of patients admitted for ACS have disturbed glucose metabolism, including diabetes, with high prevalence of previously undiagnosed dysglycaemia. Both patients with known diabetes and dysglycaemia discovered by OGTT show a high risk for poor clinical prognosis.

Keywords: Cardiovascular disease, Diabetes mellitus, Impaired glucose tolerance, Prognosis

\section{Background}

There is a global increase in the prevalence of type 2 diabetes mellitus (T2DM) and impaired glucose tolerance (IGT), which are major risk factors for atherosclerosis including acute coronary syndrome (ACS) caused by coronary artery disease (CAD) $[1,2]$. T2DM and IGT have been shown to occur among as many as two-thirds of patients with ACS and previously unknown diabetes $[3,4]$. A long-term follow-up of these patients has

\footnotetext{
*Correspondence: jeanette.kuhl@ki.se

2 Department of Medicine, Karolinska Institutet, Karolinska University

Hospital, 18288 Stockholm, Sweden

Full list of author information is available at the end of the article
}

demonstrated that these newly detected glucose disturbances also affect future prognosis [4].

The Euro Heart Survey shows that a majority of patients diagnosed with CAD including ACS have abnormal glucose regulation [5], and two earlier studies that included previously healthy individuals showed that IGT leads to an increased risk of cardiovascular disease (CVD) and death $[6,7]$. There are several factors contributing to why patients with T2DM and IGT are particularly prone to develop ACS. Among these are vascular endothelial dysfunction, disturbed platelet function, decreased fibrinolytic capacity, high levels of advanced glycation end products (AGEs), dyslipidemia, hypertension and reduced insulin sensitivity $[8-12]$. 
T2DM develops slowly through stages of early impairments of glucose metabolism. A majority of subjects who develop T2DM demonstrate IGT approximately 5 years prior to diagnosis [13]. With regard to ACS, the research to date has tended to focus on diabetes rather than IGT. Thus, the impact of IGT on ACS, and cardiovascular morbidity and mortality in general, is yet to be revealed. A large study of patients with CAD showed that diabetes, whether it is previously known or newly detected, is an independent risk factor for mortality and myocardial infarction during a 1-year follow-up period [14]. Impaired glucose regulation, including IGT and impaired fasting glucose (IFG), was not an independent predictor of adverse outcomes in these patients [14]. However, over a longer period of time, glucose levels far below the threshold for diabetes have been shown to markedly increase the risk of cardiovascular events [15].

The primary aim of the present study was to investigate the importance of dysglycaemia detected by an oral glucose tolerance test (OGTT) for long-term mortality and cardiovascular events in patients with ACS.

\section{Methods \\ Patients}

Included in the study were 1062 consecutive patients, 281 women and 781 men, aged $32-80$ years, admitted to the coronary care unit at Danderyd Hospital for myocardial infarction or unstable angina between January 19, 2006 and December 29, 2008. All patients, except patients with known type 1 or type 2 diabetes, underwent a standardised 75-g OGTT according to WHO criteria [16] at 4-5 days after admission. According to the American Diabetes Association document from 1997 [17], the criteria for IFG were a fasting plasma glucose level of $\geq 6.1$ to $<7.0 \mathrm{mmol} / \mathrm{l}$ and a 2 -h plasma glucose level at OGTT $<7.8 \mathrm{mmol} / \mathrm{l}$; and for IGT, a fasting plasma glucose level $<6.1 \mathrm{mmol} / \mathrm{l}$ and a 2-h plasma glucose level at OGTT of $\geq 7.8$ to $<11.1 \mathrm{mmol} / \mathrm{l}$. T2DM was defined as having a fasting plasma glucose value $\geq 7.0 \mathrm{mmol} / \mathrm{l}$, and/or a 2 -h plasma glucose value at OGTT $\geq 11.1 \mathrm{mmol} / \mathrm{l}$.

Myocardial infarction and unstable angina pectoris were defined according to the criteria recommended by the Joint European Society of Cardiology and the American College of Cardiology Committee [18].

\section{Protocol}

Data were collected in two steps:

1. Baseline data and OGTT results were collected at the time of admission from 2006 to 2008.

2. Retrospective review of the Swedish Web system for Enhancement and Development of Evidence-based Care in Heart Disease Evaluated According to Rec- ommended Therapies Registry (SWEDEHEART) and the Swedish Coronary Angiography and Angioplasty Registry (SCAAR) was performed in 2012.

\section{Statistical analysis}

Data are presented as mean \pm standard deviation (SD) or in percentage when categorical values. The Kruskal-Wallis test followed by Mann-Whitney's U test (Table 1) or Chi square test (Tables 1, 2, 3, 4) was performed to analyse differences in continuous variables between groups. Data shows absolute risks. Statistical significance was set at $\mathrm{p}<0.05$. Data processing was performed using IBM SPSS, version 22 .

\section{Ethical considerations}

All participants received written and oral information regarding the study and gave their written informed consent. The study protocol was approved by the local Ethics Committee at Karolinska Institutet.

\section{Results}

\section{Glucose tolerance}

Table 1 shows the distribution of women and men with ACS in all glucose tolerance groups. In total, 76 (27.0\%) women and $219(28.0 \%)$ men had NGT, and the corresponding figures for IFG/IGT were 77 (27.4\%) women and $222(28.4 \%)$ men; for patients with diabetes discovered by OGTT, numbers were $40(14.2 \%)$ women and 116 (14.9\%) men. Finally, 88 (31.3 \%) women and 224 (28.7\%) men had known diabetes at admission to the hospital. Among patients with diabetes, 31 (9.9 \%) had type 1 diabetes (data not shown).

\section{Basic characteristics and ACS diagnosis}

Table 1 shows that individuals with disturbances of glucose tolerance were older than those with NGT ( $\mathrm{p}<0.001$ ), and that patients with known diabetes were older than individuals with IFG/IGT as well as patients with diabetes discovered by OGTT $(\mathrm{p}<0.01)$. There was a significant increase in BMI in individuals with disturbances of glucose tolerance compared to subjects with NGT $(\mathrm{p}<0.01$ ), and patients with known diabetes had significantly higher BMI than individuals with IFG/ IGT $(\mathrm{p}<0.01)$. Furthermore, diastolic blood pressure was lower in patients with known diabetes compared to individuals with IFG/IGT ( $\mathrm{p}<0.001$ ), while there were no significant differences in systolic blood pressure levels between the four groups. At the time of admission, patients with known diabetes had higher serum creatinine levels $(\mathrm{p}<0.001)$ as well as higher prevalence of previous acute myocardial infarction (AMI), coronary artery bypass graft (CABG) and percutaneous coronary intervention $(\mathrm{PCI})(\mathrm{p}<0.01)$ compared to the three other 
Table 1 Basic characteristics in 1062 patients at time of admission for acute coronary syndrome

\begin{tabular}{|c|c|c|c|c|c|}
\hline & NGT & IFG/IGT & Diabetes $^{A}$ & Diabetes $^{B}$ & $\mathbf{P}$ \\
\hline No. & 295 & 299 & 156 & 312 & \\
\hline Female/male $(n)$ & $76 / 219$ & $77 / 222$ & $40 / 116$ & $88 / 224$ & NS \\
\hline Age (years) & $61.4(9.81)^{e, h, l}$ & $63.9(10.40)^{b, l}$ & $64.3(8.77)^{\mathrm{b}, \mathrm{k}}$ & $66.9(8.99)^{c, f, h}$ & $<0.001$ \\
\hline $\mathrm{BMI}\left(\mathrm{kg} / \mathrm{m}^{2}\right)$ & $25.9(3.96)^{e, i, l}$ & $27.1(3.92)^{b, k}$ & $27.8(3.81)^{c}$ & $28.5(5.00)^{c, e}$ & $<0.001$ \\
\hline Systolic blood pressure ${ }^{m}(\mathrm{~mm} / \mathrm{Hg})$ & $143.9(27.84)$ & $148.7(28.87)$ & $147.6(29.69)$ & $144.8(29.77)$ & NS \\
\hline Diastolic blood pressure ${ }^{m}(\mathrm{~mm} / \mathrm{Hg})$ & $82.9(14.26)$ & $85.4(15.49)^{\prime}$ & $83.6(13.90)$ & $80.2(15.34)^{f}$ & $<0.001$ \\
\hline S-Creatinine $(\mu \mathrm{mol} / \mathrm{L})$ & $84.0(19.95)^{\prime}$ & $86.7(33.40)^{\prime}$ & $81.9(17.44)^{\prime}$ & $111.6(89.31)^{\mathrm{c}, \mathrm{ffi}}$ & $<0.001$ \\
\hline \multicolumn{6}{|l|}{ Previous (\%) } \\
\hline Acute myocardial infarction & $14.7^{1}$ & $19.6^{\prime}$ & $12.9^{1}$ & $52.7^{\mathrm{c}, \mathrm{f}, \mathrm{h}}$ & $<0.001^{\Omega}$ \\
\hline Hypertension & $16.7^{\mathrm{f}, \mathrm{l}}$ & $25.7^{\mathrm{c}, \mathrm{i}, \mathrm{l}}$ & $16.9^{f, l}$ & $40.7^{\mathrm{c}, \mathrm{f,i}}$ & $<0.001^{\Omega}$ \\
\hline CABG & $14.6^{1}$ & $17.7^{1}$ & $14.6^{1}$ & $53.1^{\mathrm{c}, \mathrm{flh}}$ & $<0.001^{\Omega}$ \\
\hline $\mathrm{PCl}$ & $21.1^{l}$ & $17.9^{l}$ & $15.4^{\prime}$ & $45.5^{\mathrm{c}, \mathrm{fth}}$ & $<0.001^{\Omega}$ \\
\hline Smokers & $26.9^{9}$ & $26.7^{9}$ & $15.8^{\mathrm{a}, \mathrm{d}, \mathrm{j}}$ & $30.6^{9}$ & $<0.05^{\Omega}$ \\
\hline
\end{tabular}

Values are means (SD). Presented values are unadjusted

$C A B G$ coronary artery bypass graft, $P C l$ percutaneous coronary intervention

vs NGT, ${ }^{a} p<0.05,{ }^{b} p<0.01,{ }^{c} p<0.001$; vs IFG/IGT, ${ }^{d} p<0.05,{ }^{e} p<0.01,{ }^{f} p<0.001 ;$ vs Diabetes OGTT, ${ }^{g} p<0.05,{ }^{h} p<0.01,{ }^{i} p<0.001 ;$ vs known diabetes, ${ }^{j} p<0.05$,

${ }^{k} \mathrm{p}<0.01,{ }^{\prime} \mathrm{p}<0.001$

$\mathrm{m}$ Significance testing after adjustment for age, BMI, and sex, for the variables systolic blood pressure and diastolic blood pressure

$\Omega$ Chi square test

A Patients with diabetes discovered by OGTT

B Patients with known diabetes at admission

groups. Furthermore, the prevalence of smoking was less $(\mathrm{p}<0.05)$ among patients with diabetes discovered by OGTT as compared to the other groups. Table 1 also shows that the prevalence of previous hypertension was higher in patients with known diabetes compared to the other three groups $(\mathrm{p}<0.001)$, and higher in individuals with IFG/IGT compared to NGT and patients with diabetes discovered by OGTT $(\mathrm{p}<0.001)$.

Among individuals with NGT, $14.6 \%$ were diagnosed with unstable angina pectoris and 85.4\% AMI, and among individuals with IFG/IGT, $11.7 \%$ were diagnosed with unstable angina pectoris and $88.3 \%$ AMI. The corresponding numbers among patients with diabetes discovered by OGTT were 15.4 and $84.6 \%$, respectively. Among patients with known diabetes at admission to the hospital, $16.0 \%$ were diagnosed with unstable angina pectoris and $84.0 \%$ had AMI.

\section{In-hospital treatment and events}

Table 2 shows that patients with known diabetes less frequently underwent coronary angiography and PCI, and were more often treated with drug-eluting stents (DES) instead of bare metal stents (BMS). In total, 928 of 1062 patients underwent coronary angiography; 612 of 928 patients underwent PCI, and 537 of 612 patients were treated with stents, of which, 415 of 537 got DES, and 122 of 537 got BMS. Furthermore, the evaluation of the left ventricular ejection fraction (LVEF) was performed less in patients with known diabetes as compared to the other three groups. The prevalence of reduced ejection fraction was significantly higher in patients with known diabetes as compared to the other groups $(\mathrm{p}<0.001)$.

Medical treatment at hospital discharge is shown in Table 3. Aspirin, clopidogrel and statins were used less frequently in patients with known diabetes compared to individuals with NGT, IFG/IGT and patients with diabetes discovered by OGTT. Calcium antagonists, angiotensin II receptor blockers and diuretics were more frequently used among patients with known diabetes compared to the other three groups. Finally, there was no significant difference in the use of beta-blockers, while ACE inhibitors were less frequently used among individuals with NGT. Among patients with diabetes discovered by OGTT, $7.3 \%$ were started on oral antidiabetic medication at the time of discharge.

\section{Mortality and reinfarction}

Table 4 shows that there was significantly higher mortality within 30 days, 1 and 3 years in the group with known diabetes at admission compared to individuals with NGT and dysglycaemia (IFG, IGT and diabetes) discovered by OGTT (p < 0.001). The 3-year mortality among patients with type 1 diabetes was approximately $60 \%$ (data not shown). Figure 1 shows the cumulative mortality within 3 years in relation to the various glucose tolerance groups, which include NGT, dysglycaemia discovered by 
Table 2 In-hospital treatment and events in 1062 patients with acute coronary syndrome

\begin{tabular}{|c|c|c|c|c|c|}
\hline & NGT & IFG/IGT & Diabetes $^{A}$ & Diabetes $^{B}$ & $P$ \\
\hline No. & 295 & 299 & 156 & 312 & \\
\hline Female/male (n) & $76 / 219$ & $77 / 222$ & $40 / 116$ & $88 / 224$ & NS \\
\hline Coronary angiography \% & $92.5(273)^{\prime}$ & $94.0(281)^{\prime}$ & $92.3(144)^{l}$ & $73.7(230)^{c, f, i, i}$ & $<0.001$ \\
\hline \multicolumn{6}{|c|}{ Coronary angio-graphy in 928 patients } \\
\hline $\mathrm{PCl} \%$ (of 928) & $70.7(193)^{\prime}$ & $66.5(187)^{\prime}$ & $69.4(100)^{l}$ & $57.4(132)^{c, f, i}$ & $<0.05$ \\
\hline \multicolumn{6}{|l|}{$\mathrm{PCl}$ in 612 patients } \\
\hline Stent \% (of 612) & $87.0(168)$ & $87.7(164)$ & $87.0(87)$ & $89.4(118)$ & NS \\
\hline \multicolumn{6}{|l|}{ Stent in 537 patients } \\
\hline BMS \% & $81.5(137)^{\prime}$ & $82.9^{\prime}(136)$ & $81.6(71)^{\prime}$ & $60.2(71)^{\mathrm{c}, \mathrm{f}, \mathrm{i}}$ & $<0.001$ \\
\hline DES \% & $18.5(31)^{\prime}$ & $17.1^{\prime}(28)$ & $18.4(16)^{\prime}$ & $39.8(47)^{\mathrm{cf,i}, \mathrm{i}}$ & $<0.001$ \\
\hline CABG \% & $13.9(41)$ & $16.7(50)$ & $16.7(26)$ & $17.9(56)$ & NS \\
\hline LVEF evaluation \% & $83.4(246)^{\prime}$ & $83.3(249)^{\prime}$ & $87.2(136)^{l}$ & $76.0(237)^{c, f, i}$ & $<0.001$ \\
\hline Normal LVEF (>50 \%) & $74.4(183)^{\prime}$ & $72.3(180)^{\prime}$ & $67.6(92)^{\prime}$ & $50.6(120)^{c, f, i}$ & $<0.001$ \\
\hline Reduced LVEF (<50 \%) & $25.6(63)^{\prime}$ & $27.7(69)^{1}$ & $32.4(44)^{\prime}$ & $49.4(117)^{c, f, i}$ & $<0.001$ \\
\hline
\end{tabular}

Parenthesis show number of individuals

$P C l$ percutaneous coronary intervention, $B M S$ bare metal stents, $D E S$ drug-eluting stents, $C A B G$ coronary artery bypass graft, $L V E F$ left ventricular ejection fraction vs NGT, ${ }^{a} p<0.05,{ }^{b} p<0.01,{ }^{c} p<0.001$; vs IFG/IGT, ${ }^{d} p<0.05,{ }^{e} p<0.01,{ }^{f} p<0.001$; vs Diabetes OGTT, ${ }^{g} p<0.05,{ }^{h} p<0.01,{ }^{i} p<0.001 ;$ vs known diabetes, ${ }^{j} p<0.05$, ${ }^{k} p<0.01,{ }^{\prime} p<0.001$

A Patients with diabetes discovered by OGTT

B Patients with known diabetes at admission

Table 3 Medical treatment at hospital discharge in 1062 patients with acute coronary syndrome

\begin{tabular}{|c|c|c|c|c|c|}
\hline & NGT & IFG/IGT & Diabetes $^{A}$ & Diabetes $^{B}$ & $\mathbf{P}$ \\
\hline No. & 295 & 299 & 156 & 312 & \\
\hline Female/male (n) & $76 / 219$ & $77 / 222$ & $40 / 116$ & $88 / 224$ & NS \\
\hline \multicolumn{6}{|l|}{ Treatment at discharge (\%) } \\
\hline Aspirin & $97.8^{1}$ & $97.5^{1}$ & $96.7^{1}$ & $89.4^{\mathrm{c}, \mathrm{fi} i}$ & $<0.001$ \\
\hline Clopidogrel & $80.6^{1}$ & $81.1^{1}$ & $80.0^{1}$ & $66.7^{\mathrm{cf,i}, \mathrm{i}}$ & $<0.001$ \\
\hline Beta-blockers & $94.7^{1}$ & $96.1^{1}$ & $97.3^{1}$ & $95.0^{\mathrm{cf, \textrm {fi }}}$ & NS \\
\hline Calcium antagonists & $9.2^{1}$ & $14.6^{1}$ & $9.3^{1}$ & $24.6^{\mathrm{cf,fi}}$ & $<0.001$ \\
\hline ACE inhibitors & $51.2^{\mathrm{fi}, \mathrm{l}}$ & $62.6^{c}$ & $70.7^{c}$ & $63.8^{c}$ & $<0.001$ \\
\hline Angiotensin II receptor blockers & $7.8^{\mathrm{fi,l}, \mathrm{l}}$ & $12.5^{c, l}$ & $12.0^{c, l}$ & $21.9^{\mathrm{cf,fi}}$ & $<0.001$ \\
\hline Diuretics & $8.9^{f, i, l}$ & $16.8^{\prime}$ & $16.7^{1}$ & $42.5^{\mathrm{c}, \mathrm{fi} \mathrm{i}}$ & $<0.001$ \\
\hline Statins & $94.7^{1}$ & $92.9^{1}$ & $95.3^{1}$ & $88.0^{\mathrm{cf,fi}}$ & $<0.01$ \\
\hline Oral antidiabetic medication & $0^{i, 1}$ & $0^{i, 1}$ & $7.3^{c, f, 1}$ & $56.1^{\mathrm{cf,i}, \mathrm{i}}$ & $<0.001$ \\
\hline Insulin & $0^{1}$ & $0^{1}$ & $0^{1}$ & $52.7^{\mathrm{cf,fi}}$ & $<0.001$ \\
\hline Oral antidiabetic medication and Insulin & $0^{1}$ & $0^{\prime}$ & $0^{1}$ & $26.3^{\mathrm{cf,fi}}$ & $<0.001$ \\
\hline
\end{tabular}

vs NGT, ${ }^{a} p<0.05,{ }^{b} p<0.01,{ }^{c} p<0.001 ;$ vs IFG/IGT, ${ }^{d} p<0.05,{ }^{e} p<0.01,{ }^{f} p<0.001 ;$ vs Diabetes OGTT, ${ }^{9} p<0.05,{ }^{h} p<0.01,{ }^{i} p<0.001 ;$ vs known diabetes, ${ }^{j} p<0.05$, ${ }^{k} \mathrm{p}<0.01,{ }^{\prime} \mathrm{p}<0.001$

A Patients with diabetes discovered by OGTT

B Patients with known diabetes at admission

OGTT, and patients with known diabetes. The cumulative mortality within 3 years was significantly higher in patients with known diabetes compared to the other two groups.

During a follow-up time of $4.0( \pm 0.8)$ years, 86 patients $(28 \%)$ with known diabetes had reinfarction as compared to 36 patients (12\%) with NGT and 79 patients $(17 \%)$ with dysglycaemia discovered by OGTT.

\section{Discussion}

The results of the present study show that a majority of patients with ACS had dysglycaemia, in other words, 
Table 4 Patient mortality and reinfarction (281 women and 781 men)

\begin{tabular}{|c|c|c|c|c|}
\hline & NGT & IFG/IGT and diabetes ${ }^{A}$ & Diabetes $^{B}$ & $\mathbf{P}$ \\
\hline No. & 295 & 455 & 312 & \\
\hline Death within 30 days, n (\%) & $0^{c}$ & $1(0.2)^{c}$ & $15(4.8)^{a, b}$ & $<0.001$ \\
\hline Death within 1 year, n (\%) & $0^{c}$ & $9(2.0)^{c}$ & $39(12.5)^{a, b}$ & $<0.001$ \\
\hline Death within 3 years, n (\%) & $9(3.1)^{c}$ & $23(5.1)^{c}$ & $77(24.7)^{\mathrm{a}, \mathrm{b}}$ & $<0.001$ \\
\hline Reinfarction, n (\%) & $36(12.2)^{c}$ & $79(17.4)^{c}$ & $86(27.6)^{a, b}$ & $<0.001$ \\
\hline
\end{tabular}

vs NGT ${ }^{a} p<0.001$; vs IFG/IGT and diabetes OGTT $^{b} p<0.001$; vs known diabetes, ${ }^{c} p<0.001$

A Patients with diabetes discovered by OGTT

${ }^{B}$ Patients with known diabetes at admission

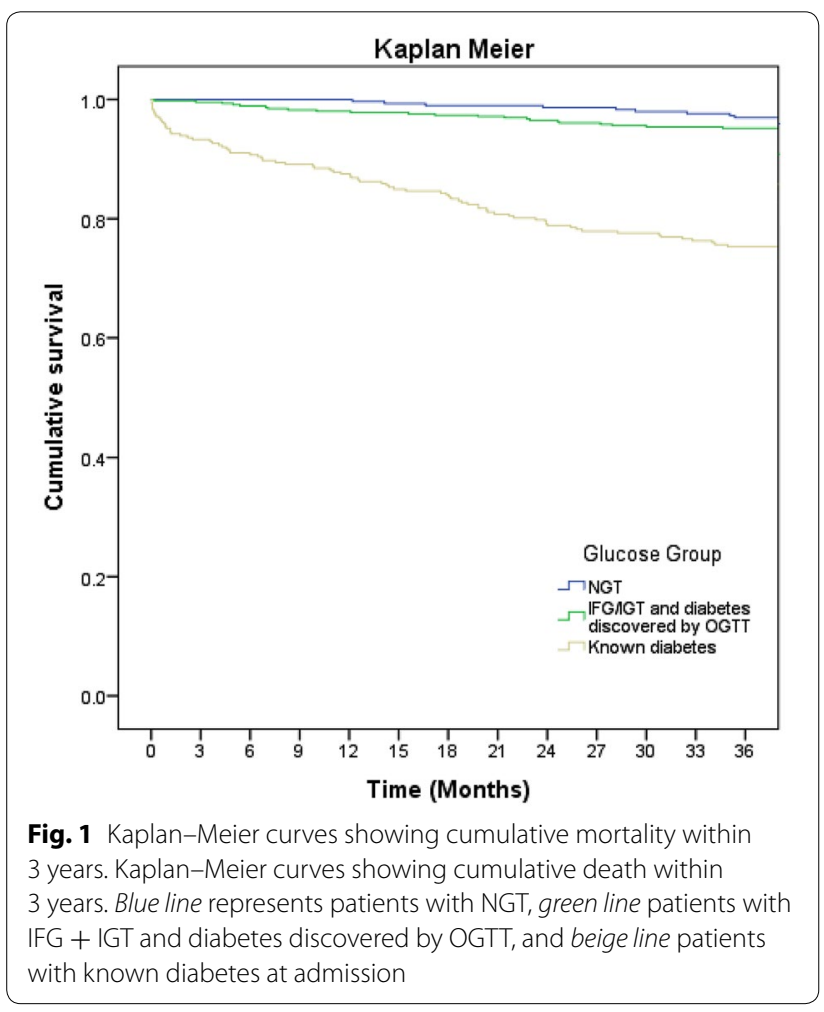

known diabetes or disturbed glucose metabolism discovered by OGTT.

Patients with known diabetes had significantly higher short- and long-term mortality as compared to both patients with NGT and those with dysglycaemia discovered by OGTT; however, the latter group showed a nonsignificant trend towards higher long-term mortality as compared to the patients with NGT.

The baseline characteristics between patients with NGT and abnormal OGTT were not significantly different, while patients with known diabetes had significantly higher co-morbidity as seen with, for example, previous myocardial infarction, higher serum creatinine and BMI, which, in addition to higher age, can at least partly explain the poor outcome in these patients. Furthermore, the prevalence of systolic left ventricular (LV) dysfunction measured by echocardiography was significantly higher in patients with known diabetes. Echocardiographic parameters such as ejection fraction and myocardial strain reflecting LV systolic function, are associated with clinical outcome in patients with myocardial infarction $[19,20]$, and LV function is one the strongest predictors of outcome following PCI [21].

We found a high incidence of previously undiagnosed diabetes and/or IFG/IGT among patients with ACS. Notably, a substantial proportion of patients who died or had myocardial infarction during the follow-up were characterized as dysglycaemic, in other words, as having known diabetes at admission or disturbed glucose metabolism diagnosed by OGTT. Interestingly, the results indicate worsened prognosis, i.e. higher mortality and reinfarction for every stage that dysglycaemia progresses, that is, a trend towards higher mortality and reinfarction in patients with positive OGTT as compared to NGT, and significantly higher mortality and reinfarction in patients with known diabetes as compared to NGT. T2DM is preceded by disturbed glucose metabolism which at least at early stages may pass undiagnosed and untreated for several years. Disturbed glucose metabolism, also at early stages, seems to have a progressive adverse effect on the cardiovascular system reflected as poor clinical outcome, most significantly in patients with manifest diabetes. Early diagnosis and treatment of impaired glucose metabolism may slow down or even reverse the adverse effects on the cardiovascular system, while irreversible damages occur when impaired glucose metabolism progresses untreated to manifest diabetes.

Previous retrospective studies have investigated the prevalence of dysglycaemia and its influence on clinical outcome in patients with CAD [22-30]. However, the accuracy of the results of some of these studies is worthy of discussion either due to the methods that were used to diagnose diabetes, which included fasting blood glucose and HbA1c levels, or inclusion of patients with 
different diagnosis of $\mathrm{CAD}$, such as, for example, stable vs ACS, associated with different short- and long-term prognosis. In the present study, we performed OGTT to investigate the glycaemic status of all patients with previously unknown DM. The European Diabetes Society and European guidelines on cardiology recommend the performance of OGTT in patients with CVD, especially in those with ACS. In patients with ACS, an abnormal OGTT seems to be a better prognostic marker than fasting blood glucose [31-34] or HbA1c alone [34].

Although the importance of hyperglycaemia as a predictor of survival in patients with ACS is well established, the association between dysglycaemia and mortality might differ across the spectrum of CAD [35]. In the present study, we included only patients with ACS, in other words, those with unstable angina and myocardial infarction.

In our study a substantial number of patients with known diabetes were treated conservatively; that is, significantly fewer patients underwent coronary angiography and PCI. The reason why a less invasive approach was chosen in the treatment of patients with known diabetes in this study is unclear. One reason could be that patients with known diabetes had already undergone coronary angiography during previous admissions since more patients in this group had previous MI, and perhaps based on previous examinations and/or decisions, a more conservative approach was chosen when the patient was readmitted with a new event. Another possible explanation concerns the risks of kidney dysfunction caused by the contrast given during coronary angiography and PCI, in other words, contrast-induced nephropathy (CIN), since the renal function was already significantly deteriorated in these patients as compared to patients in the two other groups. Higher age and existence of significant co-morbidities may also have played a role in choosing a more conservative approach. A non-invasive treatment strategy may have influenced the long-term prognosis in patients with known diabetes, as it has been demonstrated that an invasive strategy in the management of patients with ACS reduces long-term cardiovascular mortality and morbidity [36].

Although kidney dysfunction influences the likelihood of choosing an invasive treatment strategy in patients with ACS [37], previous studies have shown that an early invasive therapy is associated with better clinical outcome in patients with mild to moderate kidney dysfunction [38]. However, kidney function remains a significant prognostic marker of outcome following PCI [21]. The interrelationship between kidney dysfunction and treatment strategy as, for example, with invasive vs non-invasive approaches, in patients with diabetes and ACS has not been fully investigated.
Of note, a majority of patients with known diabetes who underwent $\mathrm{PCI}$ were treated with bare metal stents (BMS), and less than $20 \%$ of the patients with dysglycaemia detected by OGTT received drug-eluting stents (DES), since they were treated as non-diabetic patients at the time of PCI. As demonstrated in our study, a significant number of patients with positive OGTT before discharge from the hospital were actually treated as nondiabetic patients at the time of admission and, more importantly, during coronary angiography and PCI, which may have influenced the treatment strategies such as stent choice, thus possibly contributing to worse clinical outcome as seen in these patients [21]. These findings highlight the necessity of investigating glycaemic status early in patients with ACS as, for example, before coronary angiography, since it has influence on therapeutic strategies such as PCI vs CABG, choice of stent, and more. However, since this study was performed, the use of DES has increased tremendously in our department as well as all over Sweden, and currently the majority of patients, with or without diabetes, receive DES.

Due to the lack of initial symptoms, the diabetes diagnosis may be delayed for several years, even after entering a diabetic state [39]. The duration and severity of the disease are of great importance for cardiovascular risk as well as other diabetic complications. Both improved metabolic and blood pressure control has been shown to decrease morbidity and mortality in patients with CAD. Glycated haemoglobin (HbA1c) is commonly used as a measure of metabolic control. There is a strong relationship between $\mathrm{HbA1c}$ levels and mortality in patients with CAD, which is independent of other risk factors [40, 41]. In patients with ACS, high HbAlc levels are associated with worse short-term outcome [22, 42], and elevated levels of $\mathrm{HbA} 1 \mathrm{c}$ are associated with the progression and difficulty of CAD [43]. However, improved glycaemic control was not associated with a decreased incidence of macrovascular complications or mortality [44]. In the present study, there are no available data of HbA1c at the time of admission and/or during hospital stay, and no data regarding glycaemic and blood pressure control during follow-up.

Today there is no consensus among cardiologists how to treat patients with T2DM or IGT, and which glucoselowering therapy is optimal after a coronary event. One large clinical trial found that treatment with insulin in patients with myocardial infarction was associated with an enhanced risk of recurrent nonfatal myocardial infarction or stroke, while treatment with metformin was more beneficial $[45,46]$.

Another study found that insulin treatment with or without oral glucose-lowering therapy was associated with higher long-term mortality in patients with DM undergoing 
coronary angiography [47]. T2DM patients are often treated with high insulin doses leading to weight gain and high blood pressure and, despite intensified insulin therapy, the treatment goals, which also include improved metabolic control and tight blood pressure control, are not reached.

A limitation of our study is when interpreting the results one should take into consideration that this is a retrospective single-center study analysing register data lacking information regarding long-term glycaemic control and incidence of CIN during the follow-up period which are important predictors of outcome in patients with ACS. Furthermore, the register data from SWEDEHEART and SCAAR does not provide information regarding changes of medical therapy including antidiabetic treatment during the follow-up period.

\section{Conclusions}

The results of the present study show that undiagnosed dysglycaemia is common in patients with ACS. Both known diabetes and newly detected dysglycaemia showed increased risk for poor long-term clinical outcome, while short-term mortality was greater among patients with known diabetes. Routine OGTT in patients with ACS is useful for detecting undiagnosed dysglycaemia; however, new therapeutic approaches are needed to improve longterm prognosis in these patients.

\begin{abstract}
Abbreviations
ACS: acute coronary syndrome; AGEs: advanced glycation end products; BMI: body mass index; BMS: bare metal stent; CABG: coronary artery bypass graft; CAD: coronary artery disease; CIN: contrast induced nephropathy; CVD: cardiovascular disease; DES: drug-eluting stent; HbA1c: haemoglobin A1 C; IFG: impaired fasting glucose; IGT: impaired glucose tolerance; LVEF: left ventricular ejection fraction; NGT: normal glucose tolerance; OGTT: oral glucose tolerance test; PCl: percutaneous coronary intervention; SCAAR: Swedish coronary angiography and angioplasty registry; SD: standard deviation; SWEDEHEART: Swedish web system for enhancement and development of evidence-based care in heart disease evaluated according to recommended therapies registry; T2DM: type 2 diabetes mellitus.
\end{abstract}

\section{Authors' contributions}

All authors made substantial contribution to the design of the study protocol and in data analysis. The manuscript was critically revised. All authors read and approved the final manuscript.

\section{Author details}

${ }^{1}$ Division of Cardiovascular Medicine, Department of Clinical Sciences, Karolinska Institutet, Danderyd Hospital, Stockholm, Sweden. ${ }^{2}$ Department of Medicine, Karolinska Institutet, Karolinska University Hospital, 18288 Stockholm, Sweden. ${ }^{3}$ Division of Medicine, Department of Clinical Sciences, Karolinska Institutet, Danderyd Hospital, Stockholm, Sweden.

\section{Acknowledgements}

We are grateful to the steering committee of SWEDEHEART and SCAAR for providing us with data for this study. This study was supported by grants from the Swedish Society of Medicine.

\section{Compliance with ethical guidelines}

\section{Competing interests}

The authors declare that they have no competing interests.
Received: 11 June 2015 Accepted: 3 September 2015

Published online: 17 September 2015

\section{References}

1. Shaw JE, Sicree RA, Zimmet PZ. Global estimates of the prevalence of diabetes for 2010 and 2030. Diabetes Res Clin Pract. 2010;87(1):4-14.

2. Kanters SD, Banga JD, Stolk RP, Algra A. Incidence and determinants of mortality and cardiovascular events in diabetes mellitus: a meta-analysis. Vasc Med (London, England). 1999;4(2):67-75.

3. Norhammar A, Tenerz A, Nilsson G, Hamsten A, Efendic S, Ryden L, Malmberg K. Glucose metabolism in patients with acute myocardial infarction and no previous diagnosis of diabetes mellitus: a prospective study. Lancet. 2002;359(9324):2140-4.

4. Ritsinger V, Tanoglidi E, Malmberg K, Nasman P, Ryden L, Tenerz A, Norhammar A. Sustained prognostic implications of newly detected glucose abnormalities in patients with acute myocardial infarction: long-term follow-up of the Glucose tolerance in patients with acute myocardial infarction cohort. Diabetes Vas Dis Res. 2015;12(1):23-32.

5. Bartnik M, Ryden L, Ferrari R, Malmberg K, Pyorala K, Simoons M, Standl E, Soler-Soler J, Ohrvik J. The prevalence of abnormal glucose regulation in patients with coronary artery disease across Europe. The Euro Heart Survey on diabetes and the heart. Eur Heart J. 2004;25(21):1880-90.

6. DECODE Study Group. Glucose tolerance and cardiovascular mortality: comparison of fasting and 2-hour diagnostic criteria. Arch Intern Med. 2001;161(3):397-405.

7. Barr EL, Zimmet PZ, Welborn TA, Jolley D, Magliano DJ, Dunstan DW, Cameron AJ, Dwyer T, Taylor HR, Tonkin AM, et al. Risk of cardiovascular and all-cause mortality in individuals with diabetes mellitus, impaired fasting glucose, and impaired glucose tolerance: the Australian Diabetes, Obesity, and Lifestyle Study (AusDiab). Circulation. 2007;116(2):151-7.

8. Lehto S, Ronnemaa T, Haffner SM, Pyorala K, Kallio V, Laakso M. Dyslipidemia and hyperglycemia predict coronary heart disease events in middle-aged patients with NIDDM. Diabetes. 1997:46(8):1354-9.

9. Rodrigues B, Cam MC, McNeill JH. Metabolic disturbances in diabetic cardiomyopathy. Mol Cell Biochem. 1998;180(1-2):53-7.

10. Turner RC, Millns H, Neil HA, Stratton IM, Manley SE, Matthews DR, Holman RR. Risk factors for coronary artery disease in non-insulin dependent diabetes mellitus: United Kingdom Prospective Diabetes Study (UKPDS: 23). BMJ (Clinical research ed). 1998;316(7134):823-8.

11. Hardin NJ. The myocardial and vascular pathology of diabetic cardiomyopathy. Coron Artery Dis. 1996;7(2):99-108.

12. Fukushima Y, Daida H, Morimoto T, Kasai T, Miyauchi K, Yamagishi S, Takeuchi M, Hiro T, Kimura T, Nakagawa Y, et al. Relationship between advanced glycation end products and plaque progression in patients with acute coronary syndrome: the JAPAN-ACS sub-study. Cardiovasc Diabetol. 2013;12:5.

13. Unwin N, Shaw J, Zimmet P, Alberti KG. Impaired glucose tolerance and impaired fasting glycaemia: the current status on definition and intervention. Diabet Med J Br Diabet Assoc. 2002;19(9):708-23.

14. Lenzen M, Ryden L, Ohrvik J, Bartnik M, Malmberg K, Scholte Op Reimer W, Simoons ML. Diabetes known or newly detected, but not impaired glucose regulation, has a negative influence on 1-year outcome in patients with coronary artery disease: a report from the Euro Heart Survey on diabetes and the heart. Eur Heart J. 2006;27(24):2969-74.

15. Coutinho M, Gerstein HC, Wang Y, Yusuf S. The relationship between glucose and incident cardiovascular events. A metaregression analysis of published data from 20 studies of 95,783 individuals followed for 12.4 years. Diabetes Care. 1999;22(2):233-40.

16. Alberti KGMM, WHO Consultation. Diabetes mellitus. Report of a WHO Study Group. World Health Organ Tech Rep Ser. 1985;727:1-113.

17. The Expert Committee on the Diagnosis and Classification of Diabetes Mellitus. Report of the expert committee on the diagnosis and classification of diabetes mellitus. Diabetes care. 1997;20(7):1183-97.

18. Thygesen K, Alpert JS. Myocardial infarction redefined-a consensus document of The Joint European Society of Cardiology/American College of Cardiology Committee for the redefinition of myocardial infarction. Eur Heart J. 2000;21(18):1502-13. 
19. Westholm C, Johnson J, Jernberg T, Winter R. The prognostic value of mechanical left ventricular dyssynchrony in patients with acute coronary syndrome. Cardiovasc Ultrasound. 2013;11:35.

20. Moller JE, Hillis GS, Oh JK, Reeder GS, Gersh BJ, Pellikka PA. Wall motion score index and ejection fraction for risk stratification after acute myocardial infarction. Am Heart J. 2006;151(2):419-25.

21. Landes U, Kornowski R, Assali A, Vaknin-Assa H, Greenberg G, Lev El, Bental T. Predictors of long term outcomes in 11,441 consecutive patients following percutaneous coronary interventions. Am J Cardiol. 2015;115(7):855-9.

22. Giraldez RR, Clare RM, Lopes RD, Dalby AJ, Prabhakaran D, Brogan GX, Jr. Giugliano RP, James SK, Tanguay JF, Pollack CV, Jr. et al. Prevalence and clinical outcomes of undiagnosed diabetes mellitus and prediabetes among patients with high-risk non-ST-segment elevation acute coronary syndrome. Am Heart J. 2013; 65(6):918-925 e912.

23. Kosiborod M, Rathore SS, Inzucchi SE, Masoudi FA, Wang Y, Havranek EP, Krumholz HM. Admission glucose and mortality in elderly patients hospitalized with acute myocardial infarction: implications for patients with and without recognized diabetes. Circulation. 2005;111(23):3078-86.

24. Sinnaeve PR, Steg PG, Fox KA, Van de Werf F, Montalescot G, Granger CB, Knobel E, Anderson FA, Dabbous OH, Avezum A. Association of elevated fasting glucose with increased short-term and 6-month mortality in ST-segment elevation and non-ST-segment elevation acute coronary syndromes: the Global Registry of Acute Coronary Events. Arch Intern Med. 2009;169(4):402-9.

25. Kolman L, Hu YC, Montgomery DG, Gordon K, Eagle KA, Jackson EA. Prognostic value of admission fasting glucose levels in patients with acute coronary syndrome. Am J Cardiol. 2009;104(4):470-4.

26. Ergelen M, Uyarel H, Cicek G, IsikT, Osmonov D, Gunaydin ZY, Bozbay M, Turer A, Gul M, Abanonu GB, et al. Which is worst in patients undergoing primary angioplasty for acute myocardial infarction? Hyperglycaemia? Diabetes mellitus? Or both? Acta Cardiol. 2010;65(4):415-23.

27. Kuramitsu S, Yokoi H, Domei T, Nomura A, Watanabe H, Yamaji K, Soga Y, Arita T, Kondo K, Shirai S, et al. Impact of post-challenge hyperglycemia on clinical outcomes in Japanese patients with stable angina undergoing percutaneous coronary intervention. Cardiovasc Diabetol. 2013;12:74.

28. de la Hera JM, Delgado E, Hernandez E, Garcia-Ruiz JM, Vegas JM, Avanzas P, Lozano I, Barriales-Villa R, Hevia S, Martin JS, et al. Prevalence and outcome of newly detected diabetes in patients who undergo percutaneous coronary intervention. Eur Heart J. 2009;30(21):2614-21.

29. Chen PC, Chua SK, Hung HF, Huang CY, Lin CM, Lai SM, Chen YL, Cheng $\mathrm{JJ}$, Chiu CZ, Lee $\mathrm{SH}$, et al. Admission hyperglycemia predicts poorer short- and long-term outcomes after primary percutaneous coronary intervention for ST-elevation myocardial infarction. J Diabetes Investig. 2014;5(1):80-6.

30. Antonio N, Fernandes R, Soares A, Soares F, Lopes A, Carvalheiro T, Paiva A, Pego GM, Providencia LA, Goncalves $L$, et al. Reduced levels of circulating endothelial progenitor cells in acute myocardial infarction patients with diabetes or pre-diabetes: accompanying the glycemic continuum. Cardiovasc Diabetol. 2014;13:101.

31. Tamita K, Katayama M, Takagi T, Yamamuro A, Kaji S, Yoshikawa J, Furukawa Y. Newly diagnosed glucose intolerance and prognosis after acute myocardial infarction: comparison of post-challenge versus fasting glucose concentrations. Heart (British Cardiac Society). 2012;98(11):848-54.

32. Ye Y, Xie H, Zhao X, Zhang S. The oral glucose tolerance test for the diagnosis of diabetes mellitus in patients during acute coronary syndrome hospitalization: a meta-analysis of diagnostic test accuracy. Cardiovasc Diabetol. 2012;11:155.

33. de la Hera JM, Garcia-Ruiz JM, Martinez-Camblor P, Martin M, Telleria AL, Corros C, Torres F, Fernandez-Cimadevilla OC, Alvarez-Pichel I, Capin E, et al. Real incidence of diabetes mellitus in a coronary disease population. Am J Cardiol. 2013;111(3):333-8.

34. Hage C, Lundman P, Ryden L, Mellbin L. Fasting glucose, HbA1C, or oral glucose tolerance testing for the detection of glucose abnormalities in patients with acute coronary syndromes. Eur J Prev Cardiol. 2013;20(4):549-54.

35. Yang SW, Zhou YJ, Tian XF, Pan GZ, Liu YY, Zhang J, Guo ZF, Chen SY, Gao ST, Du J, et al. Association of dysglycemia and all-cause mortality across the spectrum of coronary artery disease. Mayo Clin Proc. 2013:88(9):930-41.

36. Fox KA, Clayton TC, Damman P, Pocock SJ, de Winter RJ, Tijssen JG, Lagerqvist $B$, Wallentin L. Long-term outcome of a routine versus selective invasive strategy in patients with non-ST-segment elevation acute coronary syndrome a meta-analysis of individual patient data. J Am Coll Cardiol. 2010;55(22):2435-45.

37. Szummer K, Lundman P, Jacobson SH, Schon S, Lindback J, Stenestrand $U$, Wallentin L, Jernberg T. Relation between renal function, presentation, use of therapies and in-hospital complications in acute coronary syndrome: data from the SWEDEHEART register. J Intern Med. 2010;268(1):40-9.

38. Szummer K, Lundman P, Jacobson SH, Schon S, Lindback J, Stenestrand U, Wallentin $L$, Jernberg T. Influence of renal function on the effects of early revascularization in non-ST-elevation myocardial infarction: data from the Swedish Web-System for Enhancement and Development of EvidenceBased Care in Heart Disease Evaluated According to Recommended Therapies (SWEDEHEART). Circulation. 2009;120(10):851-8.

39. Harris MI, Klein R, Welborn TA, Knuiman MW. Onset of NIDDM occurs at least 4-7 yr before clinical diagnosis. Diabetes Care. 1992;15(7):815-9.

40. Khaw KT, Wareham N, Bingham S, Luben R, Welch A, Day N. Association of hemoglobin A1 c with cardiovascular disease and mortality in adults: the European prospective investigation into cancer in Norfolk. Ann Intern Med. 2004;141(6):413-20.

41. Dale AC, Midthjell K, Nilsen TI, Wiseth R, Vatten LJ. Glycaemic control in newly diagnosed diabetes patients and mortality from ischaemic heart disease: 20-year follow-up of the HUNT Study in Norway. Eur Heart J. 2009;30(11):1372-7.

42. Kmet M, Rajer B, Pernat A. Hemoglobin A1 C is a better predictor of prognosis following the non-ST elevation acute coronary syndrome than fasting and admission glucose. Wien Klin Wochenschr. 2014;126(5-6):156-62.

43. Berry C, Noble S, Gregoire JC, Ibrahim R, Levesquie S, Lavoie MA, L'Allier $\mathrm{PL}$, Tardif JC. Glycaemic status influences the nature and severity of coronary artery disease. Diabetologia. 2010;53(4):652-8.

44. Gerstein HC, Miller ME, Genuth S, Ismail-Beigi F, Buse JB, Goff DC Jr, Probstfield JL, Cushman WC, Ginsberg HN, Bigger JT, et al. Long-term effects of intensive glucose lowering on cardiovascular outcomes. N Engl J Med. 2011;364(9):818-28.

45. Mellbin LG, Malmberg K, Norhammar A, Wedel H, Ryden L. The impact of glucose lowering treatment on long-term prognosis in patients with type 2 diabetes and myocardial infarction: a report from the DIGAMI 2 trial. Eur Heart J. 2008;29(2):166-76.

46. Vigneri R, Vigneri P, Frittitta L. Basal insulin and cardiovascular and other outcomes. N Engl J Med 2012;367(18):1761-1762; author reply 1763-1764.

47. Saleh N, Petursson P, Lagerqvist B, Skuladottir H, Svensson A, Eliasson B, Gudbjornsdottir S, Eeg-Olofsson K, Norhammar A. Long-term mortality in patients with type 2 diabetes undergoing coronary angiography: the impact of glucose-lowering treatment. Diabetologia. 2012;55(8):2109-17. 